\title{
EVI1 induces myelodysplastic syndrome in mice
}

\author{
Silvia Buonamici, Donglan Li, Yiqing Chi, Rui Zhao, Xuerong Wang, Larry Brace, Hongyu Ni, \\ Yogen Saunthararajah, and Giuseppina Nucifora
}

Department of Pathology and Cancer Center, University of Illinois at Chicago, Chicago, Illinois, USA.

\begin{abstract}
Myelodysplasia is a hematological disease in which genomic abnormalities accumulate in a hematopoietic stem cell leading to severe pancytopenia, multilineage differentiation impairment, and bone marrow (BM) apoptosis. Mortality in the disease results from pancytopenia or transformation to acute myeloid leukemia. There are frequent cytogenetic abnormalities, including deletions of chromosomes 5, 7, or both. Recurring chromosomal translocations in myelodysplasia are rare, but the most frequent are the $t(3 ; 3)(\mathrm{q} 21 ; \mathrm{q} 26)$ and the $\operatorname{inv}(3)(q 21 q 26)$, which lead to the inappropriate activation of the EVI1 gene located at 3q26. To better understand the role of EVI1 in this disease, we have generated a murine model of EVI1-positive myelodysplasia by BM infection and transplantation. We find that EVI1 induces a fatal disease of several stages that is characterized by severe pancytopenia. The disease does not progress to acute myeloid leukemia. Comparison of in vitro and in vivo results suggests that EVI1 acts at two levels. The immediate effects of EVI1 are hyperproliferation of BM cells and downregulation of EpoR and $c-M p l$, which are important for terminal erythroid differentiation and platelet formation. These defects are not fatal, and the mice survive for about 10 months with compensated hematopoiesis. Over this time, compensation fails, and the mice succumb to fatal peripheral cytopenia.
\end{abstract}

\section{Introduction}

EVI1 was first identified in the mouse as the site where integration of an ecotropic retrovirus leads to aggressive myeloid leukemia (1). The gene, later mapped to human chromosome 3 q26.2 (2), encodes a nuclear protein that interacts with corepressors and coactivators (3). EVI1 also exists as an alternative nontransforming isoform named MDS1/EVI1, which is transcribed by a distal promoter and independently regulated during differentiation (4). EVI1 contains 2 domains of 7 and 3 zinc-finger motifs that bind in vitro to DNA and repress transcription of artificial promoters (5-8). In vitro, EVI1 affects the hematopoietic differentiation of precursor bone marrow (BM) cells, leading to loss of erythropoietin (Epo) responsiveness (9); it also blocks the G-CSF-dependent differentiation of $32 \mathrm{Dcl} 3$ cells (8) and strongly upregulates the megakaryocytic differentiation of murine ES cells (10). The molecular mechanism by which EVI1 impairs hematopoietic differentiation is not known. Because EVI1 binds in vitro to an artificial promoter containing at least 9 tandem repeats of the AGATA sequence, it has been suggested that it could compete with the transcription factor GATA1 and deregulate erythroid genes (9). However, it has also been reported that EVI1 does not affect the expression of the Epo receptor (EpoR) in $32 \mathrm{Dcl} 3$ cells (9), suggesting that the role of EVI1 is more complex. Recently, a transgenic mouse model was developed using the murine Sca-1 promoter to drive the expression of EVI1 (11). Three independent founder lines were generated. The animals developed normally without signs of leukemia or myelodysplasia, but they showed a substantial reduction in the number of BM CFU erythroid-derived (CFU-E-derived) colonies.

Nonstandard abbreviations used: BM, bone marrow; BMT, BM transplantation; CFU-E, CFU erythroid; Epo, erythropoietin; EpoR, Epo receptor; MDS, myelodysplastic syndrome; MSCV, murine stem cell virus; $\mathrm{PB}$, peripheral blood; PE, phycoerythrin; RQ-PCR, real-time PCR; wbc, white blood cell.

Conflict of interest: The authors have declared that no conflict of interest exists.

Citation for this article: J. Clin. Invest. 114:713-719 (2004).

doi:10.1172/JCI200421716.
These results support the notion that inappropriately expressed EVI1 has a negative effect on erythropoiesis. EVI1 has also a strong effect on cell proliferation, and it was shown that it accelerates the cell cycle by interaction with BRG1, leading to activation of the E2F promoter (12). So far, no biological target genes regulated by EVI1 have been identified in vivo.

EVI1 is not detected in normal hematopoietic cells, and it is associated with the development of aggressive myeloid leukemia when it is inappropriately activated after rearrangements of chromosome band 3q26 $(5,13)$. The most frequent chromosomal translocations leading to EVI1 deregulation are the $\mathrm{t}(3 ; 3)(\mathrm{q} 21 ; \mathrm{q} 26)$ and the inv(3)(q21q26), which are observed almost exclusively in myelodysplastic syndrome (MDS) $(14,15)$. EVI1 is also activated in MDS without apparent rearrangements of chromosome 3 (16).

In the last few decades, a substantial increase has been noted in the incidence of MDS, a heterogeneous group of hematological disorders affecting a population with a median age of approximately 70 years. MDS is characterized by a stepwise progression of genetic abnormalities that can culminate in transformation to acute myeloid leukemia (17-23). However, as many as $40 \%$ of patients with MDS succumb to the consequences of cytopenia without leukemic transformation. Another feature of MDS besides the multilineage BM dysplasia is the paradox of BM hypercellularity associated with peripheral cytopenia.

Even though EVI1 has long been associated with MDS, the biological role of this gene in the disease is not known. To understand the significance and the effects of EVI1 in a normal hematopoietic environment, we have forced the expression of EVI1 in murine hematopoietic organs in vivo by retroviral infection of murine lineage-negative BM cells with an EVI1-expressing retrovirus and transplantation of the BM cells into lethally irradiated syngeneic recipients. We show that in vivo the constitutive expression of EVI1 recapitulates unique features of myelodysplasia such as megakaryocytic and erythroid hyperplasia, and erythroid dysplasia associated with a progressive pancytopenia that culminates in 
death. These defects are characteristic of human MDS (5, 14-16). In the reconstituted mice, this disease does not progress to acute leukemia and the EVI1-positive mice invariably succumb to their disease 10-12 months after BM transplantation. We have also analyzed the infected cells in vitro and found that, as previously reported (9), the expression of EVI1 in lineage-negative BM cells induces the impairment of Epo response, but in addition we found that EVI1 induces hyperproliferation of the hematopoietic cells and strongly delays their differentiation. We analyzed the expression of hematopoietic genes involved in erythroid differentiation and platelet formation to identify the biological pathways disrupted by EVI1 and found that, in contrast to previous reports, the EpoR and also the thrombopoietin receptor $c-M p l$ are repressed by EVI1 in vivo. These two receptors, essential for terminal differentiation of $\operatorname{rbc}(24,25)$ and platelet formation $(26,27)$, are the first in vitro targets of EVI1 that have been identified. Comparison of in vitro and in vivo results indicates that hyperproliferation, delayed differentiation, and impairment of erythroid differentiation, which occur immediately in the BM, do not appear to visibly affect the survival of mice for several months. Therefore, we propose that the EVI1-positive MDS in these mice progresses through two stages. In the first stage, EVI1 induces hematopoietic defects that can be partially compensated to allow survival. After 10-12 months, however, additional severe defects appear that include BM apoptosis, loss of cytokine responsiveness, and levels of mature rbc's and platelets that are too low to sustain life. These late effects, which cannot be compensated, lead to hematopoietic failure and death.

\section{Results}

EVI1 canses a fatal disease in mice. We used a retroviral system to constitutively express EVI1 in murine BM cells. We used the murine stem cell virus (MSCV) vector because it provides long-lasting low level of expression in vivo and contains the neomycin resistance selection marker suitable for in vitro studies (28). A diagram of the recombinant retroviral vector is shown in Figure 1A. Lineage-negative murine BM cells were infected in vitro and were injected into lethally irradiated syngeneic recipients as described in Methods. In parallel, control animals received BM cells infected with the MSCV vector. To evaluate the efficiency of infection, 70,000-100,000 infected BM cells were cultured in vitro with or without G418, and the number of colonies was determined. We consistently found that infection efficiency with the EVI1-containing retrovirus ranged between $20 \%$ and $30 \%$ and was $2.5-4$ times lower than with the empty retrovirus (80-85\% infection efficiency). Because of the large size of the EVI1 cDNA (about $4 \mathrm{~kb}$ ), a decrease of retrovirus titer and efficiency of infection was expected. To determine the extent of engraftment, we used donor mice with genotype C57BL/Ly5.2 and recipient irradiated mice with genotype C57BL/Ly5.1, and we evaluated by flow-cytometric analysis the fraction of BM cells stained by LY5.2 antibody that identifies the donor cells. The results indicated that the degree of engraftment ranged between $72.1 \%$ and $77.8 \%$. The expression of EVI1 in the BM of the infected mice was confirmed by Western blot analysis. The results (Figure 1B) show the expression of EVI1 in the BM of 2 moribund mice (lanes 4 and 5 ) and in the EVI1-containing retrovirus packaging cells used as positive control (lane 1). As negative controls, we used the emptyretrovirus packaging cells (lane 2 ) and the BM of a control mouse (lane 3). To determine the clonality of the disease, we analyzed the BM of 1 control and 2 EVI1-positive mice by Southern blot. The genomic DNA was extracted from the BM and digested with the endonuclease HindIII, which cuts once within the EVI1 cDNA. The position of the probe $(0.7 \mathrm{~kb}$ Ncol fragment) is shown in Figure 1A. The results (Figure $1 \mathrm{C}$ ) indicate that the disease is mono- or oligoclonal. A band of about $6.6 \mathrm{~kb}$ was detected in all 3 samples. This band identifies the endogenous Evil gene. To follow the development of hematopoietic disease, periodic peripheral blood (PB) counts were performed on both groups of animals. Initially, all the mice had normal blood profiles (Table 1). However, 8-12 months after BM transplantation (BMT) the mice that received EVI1-BM developed pancytopenia. At this terminal stage, the animals showed a reduction in platelet, $\mathrm{rbc}$, and white blood cell ( $\mathrm{wbc}$ ) counts, as well as in hemoglobin levels (Figure 1D and Table 1). This condi-
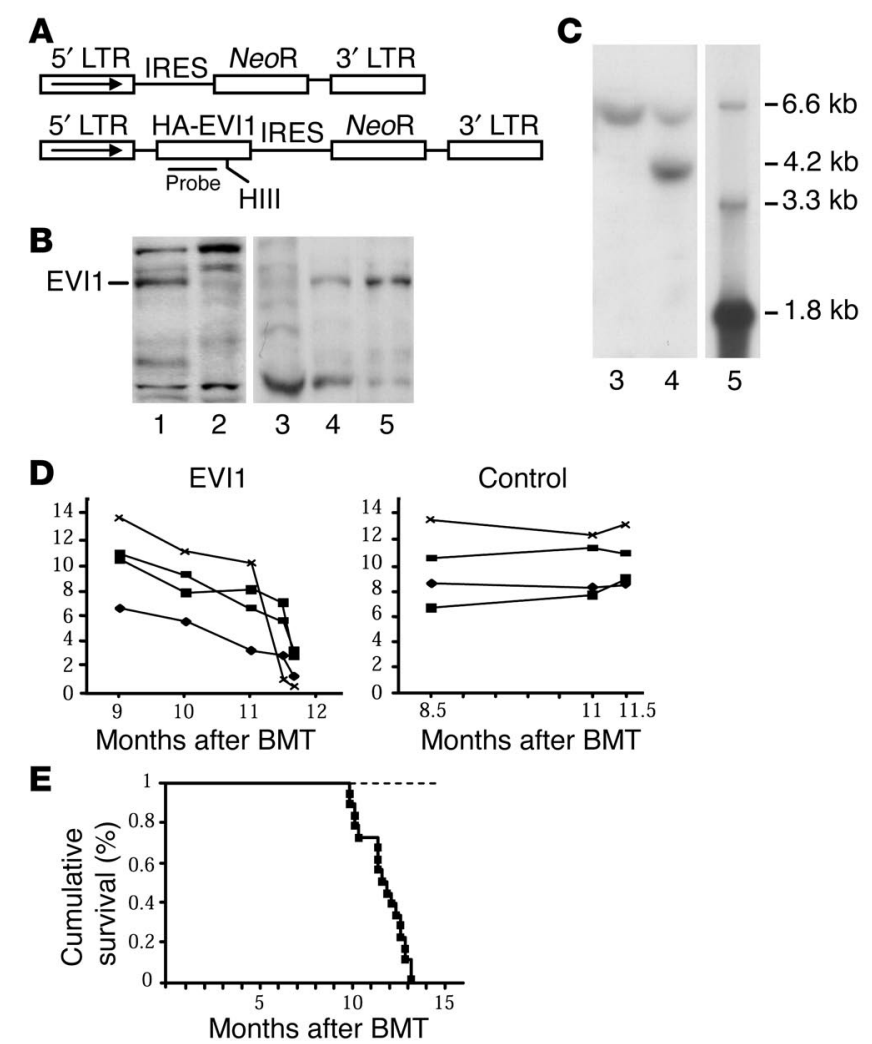

Figure 1

EVI1 causes a fatal disease in reconstituted mice. (A) Retroviral DNA constructs used in Phoenix cell line. The $5^{\prime}$ long terminal repeat (LTR) provides the promoter for a transcript that includes EVI1 and a gene encoding resistance to $\mathrm{G} 418$ (NeoR, indicated by the arrow). Internal ribosome entry site (IRES) is required for the translation of the NeoR transcript. HIII is the unique HindIII restriction site present in the EVI1 cDNA. The location of the probe used for Southern blot analysis is indicated. HA, hemagglutinin epitope. (B) Western blot analysis of EVI1-producing packaging Phoenix cells (lane 1), vector-producing packaging Phoenix cells (lane 2), control mouse BM cells (lane 3), and EVI1-positive BM cells (lanes 4 and 5) confirms the appropriate expression of EVI1 only in EVI1-positive samples (lanes 1, 4, and 5). (C) Southern blot analysis of BM cells from reconstituted EVI1-positive mice (lanes 4 and 5) and control mice (lane 3). (D) Counts of wbc's (diamonds, $\times 10^{3} / \mu \mathrm{l}$ ), rbc's (squares, $\times 10^{6} / \mu \mathrm{l}$ ), platelets (horizontal bars, $\times 10^{5} / \mu \mathrm{l}$ ), and levels of hemoglobin (crosses, $\mathrm{g} / \mathrm{dl}$ ) in PB of EVl1positive mice (left) and in control mice (right). (E) The solid line shows the Kaplan-Meier survival curve of EVI1-positive reconstituted mice. All EVI1-positive mice died or were killed because of disease conditions. The dashed line represents the survival of the control mice. 
Table 1

Hematological parameters of EVI1-positive and control mice

\begin{tabular}{|c|c|c|c|c|c|c|c|}
\hline & $\begin{array}{c}\text { Mouse } \\
\text { no. }\end{array}$ & $\begin{array}{l}\text { Latency } \\
\text { days }\end{array}$ & $\begin{array}{l}\text { wbc count } \\
\left(\times 10^{3} / \mu l\right)\end{array}$ & $\begin{array}{c}\text { HGB level } \\
(\mathrm{g} / \mathrm{dl})\end{array}$ & $\begin{array}{l}\text { rbc count } \\
\left(\times 10^{6} / \mu \mathrm{l}\right)\end{array}$ & $\begin{array}{l}\text { PLT count } \\
\left(\times 10^{3} / \mu l\right)\end{array}$ & $\begin{array}{l}\text { Spleen weight } \\
(\mathrm{mg})\end{array}$ \\
\hline EVI1 & $4^{\mathrm{A}}$ & 413 & 2.56 & 3.2 & 2.56 & 216 & 120 \\
\hline EVI1 & $11^{B}$ & 300 & ND & ND & ND & ND & ND \\
\hline EVI1 & $12^{\mathrm{A}}$ & 315 & 8.54 & 5.1 & 3.95 & 129 & 130 \\
\hline EVI1 & $14^{\mathrm{A}}$ & 308 & ND & ND & ND & ND & ND \\
\hline EVI1 & $15^{\mathrm{B}}$ & 308 & ND & ND & ND & ND & ND \\
\hline EVI1 & $17^{A}$ & 300 & ND & ND & ND & ND & ND \\
\hline EVI1 & $23^{A}$ & 353 & 7.44 & 8 & 5.68 & 120 & 80 \\
\hline EVI1 & $24^{A}$ & 345 & 2.16 & 4.8 & 3.12 & 112 & 98 \\
\hline EVI1 & $28^{A}$ & 390 & 2.79 & 6.3 & 5.67 & 243 & 60 \\
\hline EVI1 & $33^{B}$ & 345 & 1.84 & 5.6 & 4.32 & 128 & ND \\
\hline EVI1 & $34^{\mathrm{B}}$ & 360 & 11.52 & 1.6 & 0.48 & 56 & 88 \\
\hline EVI1 & $48^{A}$ & 368 & 0.85 & 5 & 2.85 & 245 & 64 \\
\hline EVI1 & $52^{\mathrm{A}}$ & 390 & 5.95 & 8 & 5.25 & 165 & ND \\
\hline EVI1 & $54^{\mathrm{A}}$ & 345 & 1.68 & 8.8 & 3.25 & 96 & ND \\
\hline EVI1 & $56^{\mathrm{A}}$ & 368 & 1.04 & 3.2 & 2.72 & 248 & 123 \\
\hline EVI1 & $67^{A}$ & 383 & 6.0 & 8.5 & 6 & 150 & 114 \\
\hline EVI1 & $75^{\mathrm{A}}$ & 383 & 4.65 & 9 & 6.55 & 495 & 43 \\
\hline EVI1 & $97^{c}$ & NA & 11.90 & 12.0 & 8.50 & 1,180 & 68 \\
\hline EVI1 & $87^{c}$ & NA & 8.26 & 11.9 & 8.33 & 1,001 & 71 \\
\hline EVI1 & $65^{c}$ & NA & 13.32 & 14.7 & 10.14 & 1,197 & 74 \\
\hline Control & $20^{D}$ & NA & 7.84 & 16 & 10.2 & 1,344 & 69 \\
\hline Control & $39 \mathrm{D}$ & $N A$ & 8.96 & 10.4 & 9.4 & 1,480 & ND \\
\hline Control & $36^{D}$ & NA & 9.68 & 12 & 8.7 & 2,544 & 73 \\
\hline Control & $35^{\mathrm{D}}$ & NA & 13.63 & 13.1 & 10.1 & 1,350 & 70 \\
\hline
\end{tabular}

HGB, hemoglobin; ND, not determined; NA, not applicable; PLT, platelet. Normal values: wbc, $3.2 \times 10^{3} / \mathrm{ul}$ $12.7 \times 10^{3} / \mathrm{ul}$; HGB, 11.8-14.9 g/dl; rbc, $7.0 \times 106 / \mathrm{ul}-10.1 \times 10^{6} / \mathrm{ul}$; PLT, $766 \times 10^{3} / \mathrm{ul}-1,657 \times 10^{3} / \mathrm{ul}$. AKilled because of moribund condition. ${ }^{B}$ Died of disease. CKilled 3 months after BMT. DNo disease.

of cell death. To confirm that apoptosis was the major mechanism of cell death, we stained spleen sections of control and EVI1-positive mice with cleaved caspase-3 antibody (Figure 2, G and $\mathrm{H}$ at $\times 10 \mathrm{mag}$ nification, and $\mathrm{I}$ and $\mathrm{J}$ at $\times 40$ magnification). The results clearly show positive immunostaining in sections of the EVI1-positive spleen (Figure 2, H and J) but not in sections of the control spleen (Figure 2, G and I). These results confirm that apoptosis rather than hemolysis is the major cause of cell death.

The control BM biopsy specimen showed trilineage hematopoiesis with normal megakaryocytes, myeloid cells, and erythroid precursors (Figure 2, K and $\mathrm{L}$ ). Trilineage hematopoiesis was also observed in the BM of EVI1-positive mice. However, the BM appeared hypercellular (Figure $2 \mathrm{M}$ ), with erythroid and megakaryocytic hyperplasia. Dyserythropoiesis as manifested by nuclear irregularity and nuclear budding of erythroid precursors was present in EVI1-positive mice (Figure $2 \mathrm{~N}$, yellow arrows). $\mathrm{PB}$ smears from EVI1-positive mice were obtained every 1-2 months and compared to smears from age-matched control mice. For approximately 7-8 months after transplantation, the $\mathrm{PB}$ morphology appeared normal (data not

tion rapidly worsened, eventually leading to death 10-12 months after BMT (Figure 1E). None of the animals progressed to acute leukemia, and we attribute the cause of death to pancytopenia.

EVI1 induces multilineage hematopoietic defects. Postmortem examination of the organs showed no marked difference in the size or appearance of the kidneys, liver, thymus, lungs, or heart. The spleens of all diseased animals showed marked congestion. About half of the diseased mice suffered from splenomegaly; however, a few mice had normal-sized spleens (Table 1). Morphological analysis of tissue paraffin sections of liver and heart tissue showed no abnormality (data not shown). In the control spleen, well-defined white pulp containing lymphocytes and red pulp were preserved as expected (Figure 2A). In contrast, the white pulp of diseased spleens appeared dispersed and the red pulp was expanded (Figure 2B). Furthermore, the red pulp showed increased numbers of erythroid precursors compared with normal controls (Figure 2, C and D). Prussian blue staining revealed large depositions of iron in BM (not shown) and spleen sections (Figure 2F) of the EVI1-positive mice. The iron depositions were not observed in the organs of control mice (Figure 2E). Increased iron depositions suggest increased rbc destruction due to either hemolysis or apoptosis. To rule out the possibility of hemolysis, we measured the total and direct bilirubin levels in the serum of normal and EVI1-positive mice. This assay has been used by other investigators to distinguish between hemolysis and apoptosis in murine studies (29). We found that the level of total and direct bilirubin in the serum of the EVI1-positive mice was not statistically different from the control sera (data not shown), suggesting that hemolysis is not the dominant cause shown). However, PB from EVI1-positive moribund mice revealed pancytopenia with marked anisopoikilocytosis and increased numbers of polychromatophilic rbc's (compare normal PB smear, Figure 2O, with EVI1-positive PB smear, Figure 2, P and Q), and presence of Howell-Jolly bodies (Figure 2R).

EVI1 represses in vitro growth of BM from EVI1-positive mice. Even though moribund EVI1-positive mice consistently had very low PB counts, their BM appeared hypercellular and contained iron deposition; this result, combined with normal bilirubin levels and caspase- 3 activation, strongly suggests that apoptosis is the cause of cell death. This apparently contradictory coexistence of hypercellular BM, cell death, and overall pancytopenia is a puzzling feature of human MDS. To determine whether EVI1 impairs the response of BM precursors to differentiation cytokines, we plated equal numbers of lineage-negative BM cells obtained from moribund EVI1-positive mice and from age-matched control mice in either Epo or GM-CSF as growth factor, and colonies were counted after 3 days (Epo) or 7 days (GM-CSF). The results show that EVI1-positive BM cells form a significantly lower number of colonies than do control cells (Figure 3A). To determine whether, in addition to the presence of EVI1, other defects must occur in the hematopoietic organs of the EVI1-positive mice to repress BM growth in vitro, we freshly infected lineage-negative normal BM cells with empty retrovirus or EVI1-containing retrovirus and plated an equal number of selected cells with either Epo or GM-CSF. The colonies and cells were counted after 3 days (Epo) and 7 days (GM-CSF). The results (Figure $3 \mathrm{~B}$ ) confirm that EVI1 has an immediate repressive effect on the growth of erythroid colonies as previously reported 

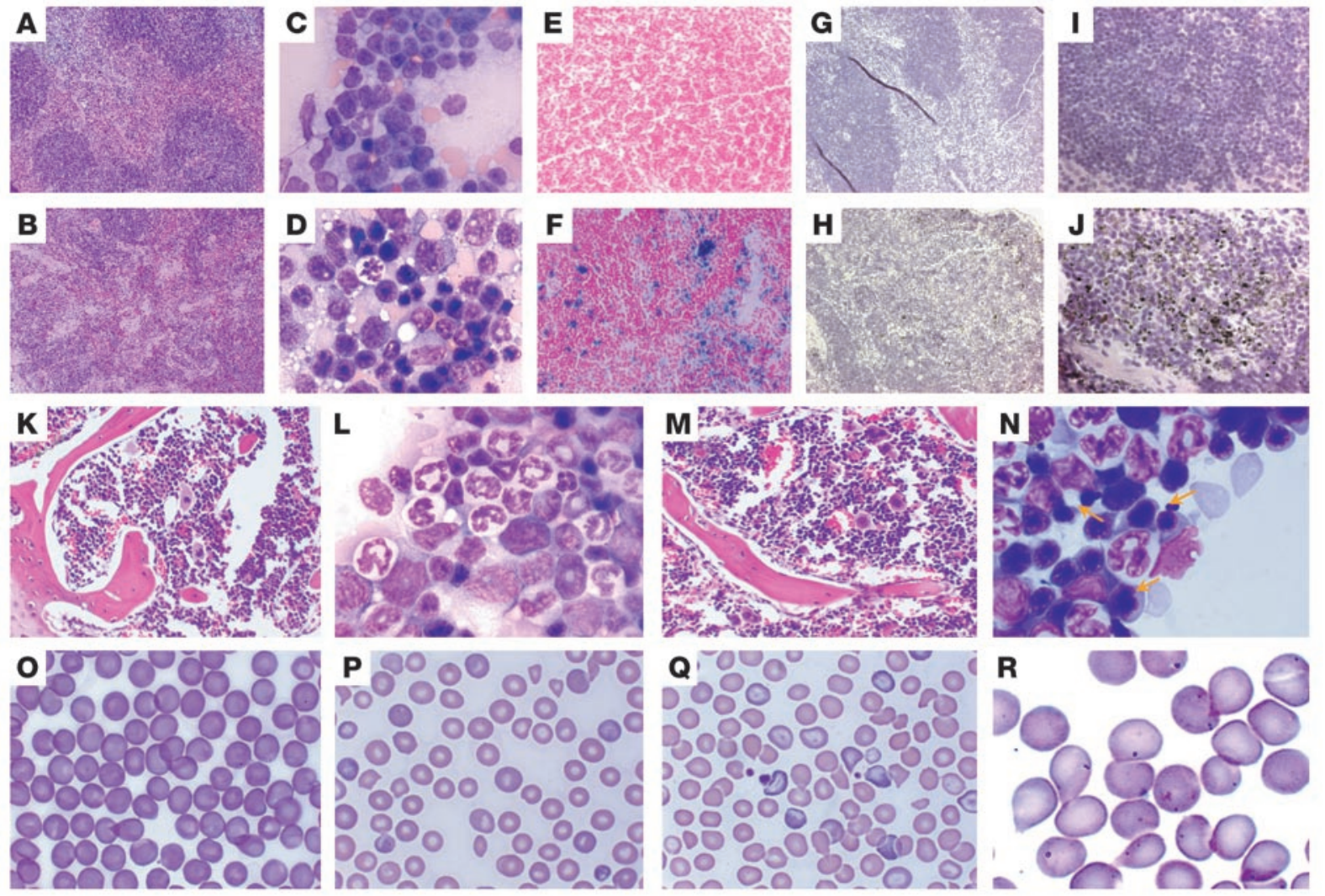

\section{Figure 2}

EVI1 induces BM hypercellularity, dyserythropoiesis, erythroid and megakaryocytic hyperplasia, and apoptosis. Sections of normal spleen tissue (A and C) show normal white and red pulp. In comparison, EVI1-positive spleen tissue shows an expansion of red pulp and erythroid hyperplasia (B and dark cells in D). (E) Prussian blue iron staining of normal spleen tissue does not identify extensive iron deposition. (F) Iron depositions are evident in a section of EVI1-positive spleen tissue stained with Prussian blue. (H and $\mathbf{J})$ Sections of EVI1-positive spleen tissue stained with cleaved caspase-3 antibody demonstrate the presence of apoptosis. ( $\mathbf{G}$ and $\mathbf{I})$ Normal spleen stained with cleaved caspase-3 antibody. (K and $\mathbf{L}$ ) Control BM biopsy specimen shows normal cellularity and trilineage hematopoiesis. (M) In contrast, the EVI1-positive BM appears hypercellular, with erythroid and megakaryocytic hyperplasia. The BM dyserythropoiesis in EVI1-positive BM aspirates is shown (N), where the arrows point to nuclear irregularity and nuclear budding of erythroid precursors. (O) A PB smear of a control mouse is shown. In contrast, the PB smear of an EVI1-positive mouse shows anisopoikilocytosis (P), increased number of polychromatophilic rbc's $(\mathbf{Q})$, and Howell-Jolly bodies (R). Magnification, $\times 10(\mathbf{A}, \mathbf{B}, \mathbf{E}, \mathbf{F}, \mathbf{G}, \mathbf{H}, \mathbf{K}, \mathbf{M}) ; \times 40(\mathbf{C}, \mathbf{D}, \mathbf{I}, \mathbf{J}, \mathbf{L}, \mathbf{N}, \mathbf{O}, \mathbf{P}, \mathbf{Q}) ; \times 100$ (R).

(9). However, if GM-CSF is added to the culture medium instead of Epo, then EVI1 induces a marked increase in the number and size of the colonies, indicating that in response to GM-CSF, EVI1 not only doubles the proliferation rate of the cells but also doubles the clonogenicity of the hematopoietic progenitors. To determine whether EVI1 impairs the response to Epo in the early stages of reconstitution, lineage-negative BM cells were isolated from EVI1positive mice and controls 3 months after transplantation. At this time, the EVI1-positive mice did not show visible signs of disease and their PB appeared normal (Table 1 and data not shown). As shown in Figure 3C, at this point in time there was already a significant reduction in the number of colonies stimulated by Epo, indicating that impairment of Epo response is an early effect of EVI1 in vivo as well as in vitro and that it occurs in the absence of obvious PB dyserythropoiesis. To determine whether EVI1 affects the differentiation of freshly infected BM, control and EVI1-positive colonies grown in GM-CSF were isolated and sequentially replated. At each step cytospin preparations were made to evaluate cell differentiation. The results confirm that in freshly infected BM cells EVI1 enhances colony formation (data not shown). Furthermore, EVI1 considerably delays the terminal differentiation of the cells from about 28 days (controls) to about 49 days. After 28 days in culture, the control cells appear differentiated, and only macrophages, characterized by small compact nuclei and large vacuolated cytoplasm, were observed (Figure 3D). In contrast, after 28 days in culture, the EVI1 cells appeared small with scarce cytoplasm and large nuclei, indicative of a more immature state (Figure 3D).

Significant increase of the erythroid marker Ter119 in EVI1-positive cells. To characterize the hematopoietic cell populations of the EVI1-positive mice, we analyzed lineage-specific antigens in BM and spleen cells of 3 moribund EVI1-positive mice and 2 control mice by flow cytometry. We tested CD3, CD4, and CD8 T cells, as well as CD45R B cells, CD18 macrophages, Ter119 erythroid cells, LY-6G granulocytes, and CD34 early hematopoietic progenitors. The percentages of most cell lineages in control and EVI1-positive mice were comparable. However, the populations of Ter119-positive cells were consistently 2 - and 3-fold higher in the spleens and $\mathrm{BM}$ of EVI1-positive mice, respectively (Figure 3E shows representative results for 1 EVI1-positive mouse and 1 control mouse). We used RT-PCR to confirm that the Ter119-positive cells express 

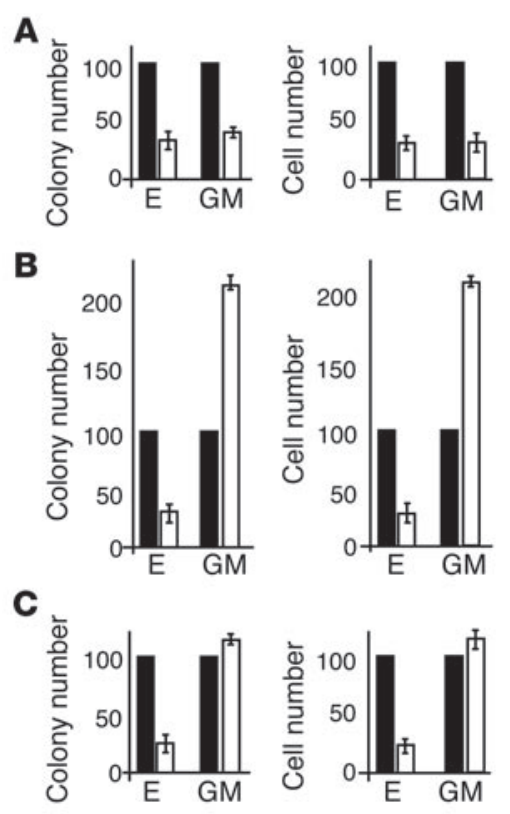

D
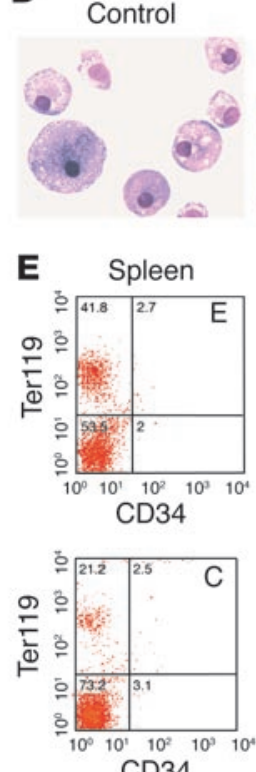

CD34
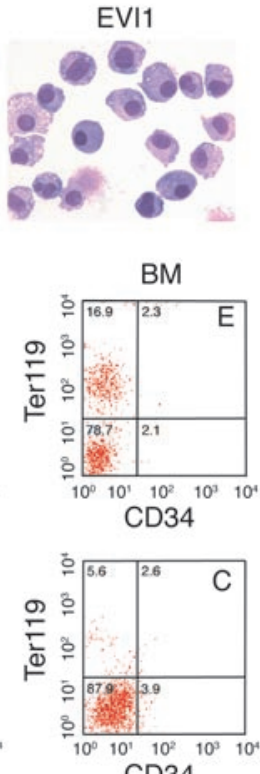

$\mathbf{F}$

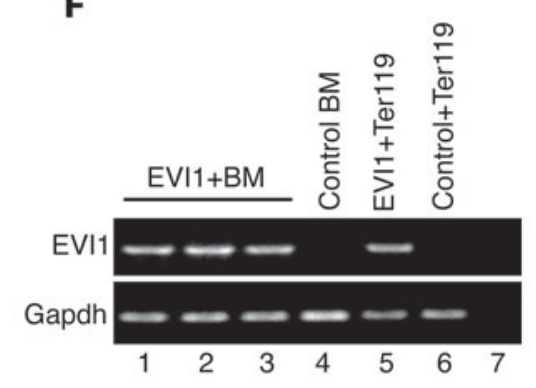

\section{Figure 3}

EVI1 alters the response to cytokines and significantly increases the number of immature erythroid cells. (A-C) A total of 15,000 lineage-negative cells were isolated from control mice BM (black bars) or BM cells of moribund EVI1-positive mice (white bars). The cells were plated in duplicate in methylcellulose and were cultured with Epo (E) or GM-CSF (GM). After 3 days (Epo) or 7 days (GM-CSF) in culture, the colonies (left panels) and the cells (right panels) in each plate were isolated and counted. (A) The decrease in the number of colonies and cells of EVI1-positive mice shows that the BM cells of these animals have impaired in vitro differentiation. (B) The same assay was carried out with lineage-negative normal BM cells freshly infected with empty retrovirus (black bars) or EVI1-containing retrovirus (white bars). In contrast to the cells obtained from the moribund mice, EVI1 represses only the response to Epo in freshly infected BM cells. (C) The same assay was carried out with lineage-negative BM cells isolated from EVI1-positive mice 3 months after transplantation (white bars) or from age-matched controls (black bars). EVI1 represses only the response to Epo. (D) Cytospin preparations of control murine BM cells (left) or BM cells of moribund EVI1-positive mice (right) stained with Wright-Giemsa stain show that EVI1 delays in vitro differentiation as indicated by the smaller size and larger, less compact nuclei of the cells. (E) The spleens and BM of EVI1-positive mice (E, top panels) have a higher number of Ter119-positive cells than the organs of a control animal (C, bottom panels). Cells were stained with Ter119-PE and CD34-FITC. The percentage of positive cells for each quadrant is noted in the upper left corner of the quadrants. (F) RT-PCR analysis shows the expression of EVI1 in total BM cells of 3 moribund mice (lanes 1,2, and 3), but not in the BM of a control mouse (lane 4). Analysis of sorted Ter119-positive cells of a moribund mouse (lane 5) confirms the expression of EVI1. EVI1 was not detected in Ter119-positive cells of the control (lane 6). For lane 7, no cDNA was added to the reaction. Gapdh was used as an internal standard.

EVI1. The results indicate that the Ter119-positive cells sorted from the EVI1-positive moribund mice, but not those sorted from the control mice, express EVI1 (Figure 3F). To determine whether the increased proliferation of erythroid cells in the spleen and BM appeared early after transplantation, we repeated the flow cytometry quantitation with the BM of EVI1-positive mice 4 months after reconstitution. At this time, the PB smears of these mice appeared normal (data not shown); however, the number of immature erythrocytes was 3-fold higher than in control animals (data not shown). The back-gating analysis of the cells positive for Ter119 localized them within a population of cells with low forward scatter (cell size) and low side scatter (cell complexity).

EpoR and c-Mpl expression is lower in EVI1 positive mice. Impairment of erythropoiesis and platelet formation are two obvious defects that are consistently observed in PB of the moribund EVI1-positive mice and that contribute to their death. To identify the differentiation steps disrupted by EVI1 in these two lineages, we used semi-quantitative RT-PCR to evaluate the expression of potential genes that regulate terminal erythropoiesis and megakaryopoiesis. We found by RT-PCR that two of them, EpoR and $c-M p l$, essential to erythroid differentiation and platelet formation (24-27), were repressed (data not shown). We used real-time PCR (RQ-PCR) to quantify more accurately the expression of EpoR and $c-M p l$ in the BM of 12 moribund EVI1-positive mice and 4 age-matched control mice (Figure 4). The data were normalized to the endogenous expression of Gapdh. The median normalized level of EpoR and c-Mpl in control samples was 231 for EpoR (range 213-244) and 216 for $c-M p l$ (range 191-224), whereas in the BM of the moribund EVI1-positive mice the level the EpoR was 102 (range 6-170) and that of $c-M p l$ was 70 (range 24-91). We found that animals with low expression of EpoR also had low expression of $c-M p l$ and vice versa. The repression of these two genes was also clearly observed 4 months after BMT at a time when the mice appeared normal, as did their PB morphology and profile (data not shown). At 4 months after BMT the median normalized level of EpoR in the BM was 108 (range 98-115), and the level of $c-M p l$ was 41 (range 39-51) (Figure 4).

\section{Discussion}

Among the hematopoietic malignancies, MDS is perhaps the least studied, in part because of the difficulty of obtaining a suitable model. We report on a murine model of EVI1-positive MDS that we have generated by BM infection and transplantation. After a relatively long latency, the reconstituted animals develop a fatal pancytopenia, accompanied by hypercellular BM and severe dys- 

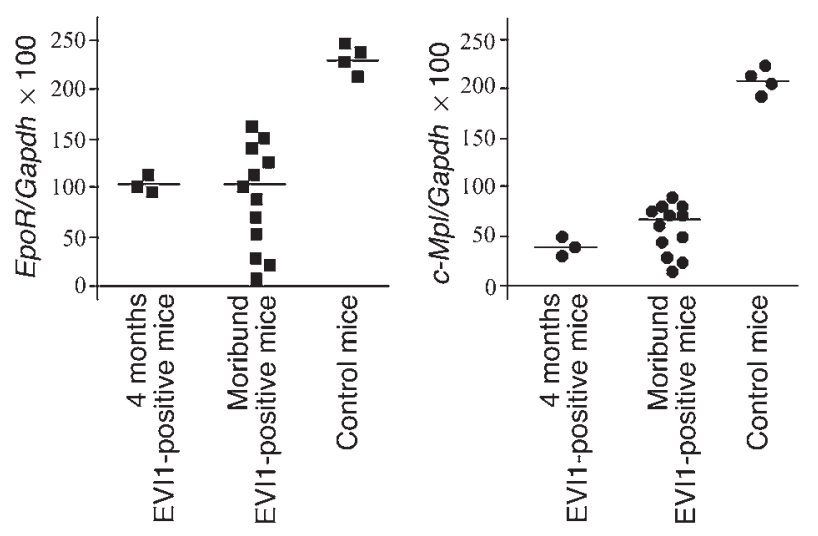

Figure 4

EVI1 represses the expression of EpoR and $c-M p l$. Real-time quantification of EpoR (squares) and c-Mpl (circles) normalized to Gapdh shows a significant decrease of these genes' median expression in the BM of EVI1-positive mice 4 months after BMT or at the time of their disease-induced death. The results are plotted as the ratio between EpoR or c-Mpl and Gapdh multiplied by 100.

erythropoiesis. These features resemble those reported in human EVI1-positive MDS (14-16). We have also determined that among the genes responsible for terminal differentiation of erythroid lineage and platelet formation, EpoR and $c-M p l(24-27)$ are repressed in the BM of the diseased mice. The repression of these receptors does not require cooperating mutations but is observed as early as 4 months after BMT, at a time when the mice behave normally and the morphology and profile of their PB are normal. It was earlier reported that inappropriately expressed EVI1 blocks erythropoiesis by binding to the DNA consensus sequence of the transcription factor GATA1 and by repressing genes regulated by GATA1 (9). Surprisingly, however, it was also reported that the expression of the GATA1-regulated EpoR was not altered (9). In contrast, by RQ-PCR we found that the expression of this gene as well as that of $c-M p l$ is strongly downregulated in the BM of EVI1positive mice. As previously suggested, it is quite probable that disruption of GATA1 functions is responsible for the downregulation of these two genes. However, the mechanism involved is probably more complex than straight DNA binding by EVI1 because, in contrast to probes that contain 9 GATA repeats (10), electrophoretic mobility shift assays show that a canonic DNA probe that specifically binds to GATA1 fails to interact with EVI1 (data not shown). We find that, in vitro, EVI1 affects normal hematopoiesis in many contradictory ways. Whereas it downregulates erythroid colony formation, it very strongly upregulates the rate of cell proliferation in response to GM-CSF, while simultaneously doubling the differentiation time. These defects appear immediately in the isolated BM cells after expression of EVI1, and they occur early in the transplanted animals as well. On the other hand, because the mice survive and behave normally for about 9 months, it is improbable that these defects by themselves cause their death. On the basis of the $\mathrm{BM}$ analysis 3 months after transplantation, we propose that, as shown in vitro, EVI1 expression in murine hematopoietic progenitors induces erythroid differentiation impairment. Because the differentiation impairment is only partial and EVI1 cells proliferate in response to cytokines, in vivo homeostatic mechanisms are probably able to prevent the development of anemia in the mice for 8-10 months, presumably through the increased hematopoietic proliferation induced by EVI1, which was evident upon morphological examination of the BM and spleen biopsy specimens. Eventually, however, progressive cytopenia occurred in the mice. Hematopoietic cells isolated from the mice at this point demonstrated impaired colony formation potential in response to cytokines in vitro, in contrast to freshly infected EVI1 hematopoietic cells and cells 3 months after BMT, which had an impaired response only to Epo. The reason for this late-onset loss of hematopoietic stem cell functional capacity may be accumulation of genetic damage during the 10 -month period of replicative stress or as a consequence of EVI1-induced genomic instability. In summary, disease progression in EVI1-positive mice demonstrates that hypercellular marrow with peripheral cytopenia, a feature of MDS, can result from specific molecular events that impair (without completely preventing) terminal differentiation. The EVI1positive mice that we generated are a suitable platform in which to test the efficacy of and optimal timing for therapeutic strategies.

\section{Methods}

Cell culture and BMT. The maintenance, transfection, and culture of the retroviral packaging cell line Phoenix (ATCC) has been reported (30). The infection, selection, and culture of murine BM progenitors were carried out as described (30). The infected BM cells were injected into lethally irradiated syngeneic recipient animals as described (31). All animal studies were performed in accordance with the guidelines of the Animal Care Committee of the University of Illinois at Chicago.

$P B$ cell count and hematocrit measurements. PB counts of recipient mice were recorded starting 4 months after transplantation. Every 2 weeks for the first 3 months, and then again once a week until the animal was killed or died of its disease, $20 \mu \mathrm{l}$ of retro-orbital PB were obtained from each animal. The blood was diluted in $3 \mathrm{ml}$ of Isoton II, and counts were obtained by using the Coulter Counter Z1 (Beckman Coulter Inc.).

Histological examination and cytochemistry. Organs and tissues were first fixed in PBS with 4\% paraformaldehyde and processed for paraffin-embedded sectioning at $5 \mu \mathrm{m}$ in thickness, then stained with one of the following as required: H\&E, Wright-Giemsa stain, white Wright-Giemsa stain, or Prussian blue. For cytochemistry, BM or spleen biopsy specimens were treated with kits from Sigma-Aldrich. PB was stained with Diff-Quik Stain Set (Dade Behring Inc.) for identification of cell morphology. For caspase staining, we used the Envision kit by DakoCytomation.

Flow cytometry immunophenotyping assay. BM or spleen cells were washed and incubated in ice for 30 minutes with $1 \mu \mathrm{l}$ of rat monoclonal anti-mouse antibody labeled with FITC, phycoerythrin (PE), or Tricolor (TC), from Caltag Laboratories and BD Biosciences - Pharmingen. The following antibodies were used: Ter119-PE, CD34-FITC, CD3-FITC, CD4-PE, CD8-TC, CD18FITC, CD45R-PE, and Gr1-TC. Cells ( $10^{4}$ per sample) were analyzed on a FACS Vantage flow cytometer (BD). Gating was based on unstained cell populations and isotype matching control antibodies. Three independent experiments were performed. The data were analyzed with CellQuest (BD) and Summit (DakoCytomation) software.

RT-PCR and RQ-PCR analysis. Total cellular RNA was extracted as described (32). The RNA was treated with DNaseI as described in the Invitrogen handbook (Invitrogen Corp.). cDNA was prepared according to the First Strand cDNA Synthesis Kit protocol (Fermentas). RT-PCR was performed in $15-\mu l$ reactions containing $1.5 \mu \mathrm{l}$ of cDNA, $0.1 \mu \mathrm{l} \mathrm{Taq}$ Red DNA Polymerase (CLP), and $300 \mathrm{nM}$ forward and reverse primers. The primers for MSCV-EVI1 were as follow: MSCVneo forward, 5'-GCATCGTTCTGTGTTGTCT-3'; EVI1-756 reverse, 5'-CGGGCATGCATGGGCCCGGGCACCGA-3'. RQ-PCR was performed in a $25-\mu \mathrm{l}$ reaction containing $5 \mu \mathrm{l}$ of cDNA, $12.5 \mu \mathrm{l}$ of the Platinum 
Quantitative PCR SuperMix-UDG (Invitrogen Corp.), $300 \mathrm{nM}$ forward and reverse primers, and $150 \mathrm{nM}$ TaqMan probe (32). The primers and probe for murine Gapdh were purchased (Applied Biosystems). The primers and probes used for the EpoR and $c-M p l$ genes were as follow: EpoR forward, $5^{\prime}$ AGTGAGCATGCCCAGGACA-3'; EpoR reverse, 5'-AAGTCAGACGGGCAGGAAGAT-3'; EpoR probe, 5'-TGGATAAGTGGTTGCTGCCCCGG-3'; c-Mpl forward, 5'-TGAACTGCTTCTCCCAAACATTT-3'; c-Mpl reverse, 5'-CACACATACCGGGGTTCCAAAG- $3^{\prime}$; $c$ - $M p l$ probe, $5^{\prime}$-GGAATACAGGGGGCATGCACGG-3'. The probes were labeled by a 5'-FAM reporter and a 3'-TAMRA quencher. All the primers and probes were designed using Primer Express software (Applied Biosystems). RQ-PCR amplification was performed on the iCycler iQ detection system, and the data were collected and analyzed using iCycler iQ version 3.0 software (Bio-Rad Laboratories Inc.).

DNA cloning, Western blot, and Southern blot analysis. EVI1 was cloned in the EcoRI and BamHI sites of the MSCV vector (BD Biosciences - Clontech). All cloning junctions were verified by DNA sequencing. Western blot was carried out as described $(3,30)$. Southern blot analysis was performed as previously described (33).

\section{Acknowledgments}

We thank John Anastasi for useful discussions. This work was supported by NIH grants NIH-NCI CA89622 and NIH-NCI CA67189 (to G. Nucifora) and by a Translational Research Award from the Leukemia \& Lymphoma Society (to G. Nucifora).

Received for publication March 25, 2004, and accepted in revised form July 6, 2004.

Address correspondence to: Giuseppina Nucifora, Department of Pathology and Cancer Center, University of Illinois at Chicago (M/C 737), 900 S. Ashland Avenue, Chicago, Illinois 60607, USA. E-mail: nucifora@uic.edu.
1. Mucenski, M.L., Taylor, B.A., Copeland, N.G., and Jenkins, N.A. 1988. Chromosomal location of EVI1 in the DNA of AKXD murine myeloid tumors. Oncol. Res. 2:219-233.

2. Morishita, K., Parker, D.S., Mucenski, M.L., Copeland, N.G., and Ihle, J.N. 1988. Retroviral activation of a novel gene encoding a zinc finger protein in IL-3-dependent myeloid leukemia cell lines. Cell. 54:831-840.

3. Chakraborty, S., Senyuk, V., Sitailo, S., Chi, Y., and Nucifora, G. 2001. Interaction of EVI1 with cAMP-responsive element-binding protein-binding protein (CBP) and $\mathrm{p} 300 / \mathrm{CBP}$-associated factor $(\mathrm{P} / \mathrm{CAF})$ results in reversible acetylation of EVI1 and in co-localization in nuclear speckles. J. Biol. Chem. 276:44936-44943.

4. Fears, S., et al. 1996. Intergenic splicing of MDS1 and EVI1 occurs in normal tissues as well as in myeloid leukemia and produces a new member of the PR family. Proc. Natl. Acad. Sci. U. S. A. 93:1642-1647.

5. Buonamici, S., Chakraborty, S., Vitalyi, S., and Nucifora, G. 2003. The role of EVI1 in normal and leukemic cells. Blood Cells Mol. Dis. 31:206-212.

6. Delwel, R., Funabiki, T., Kreider, B.L., Morishita, K., and Ihle, J.N. 1993. Four of the seven zinc fingers of the EVI1 myeloid-transforming gene are required for sequence-specific binding to GA(C/T)AAGA(T/ C)AAGATAA. Mol. Cell. Biol. 13:4291-4300.

7. Funabiki, T., Kreider, B.L., Morishita, K., and Ihle, J.N. 1990. The carboxyl domain of the zinc finger of EVI1 myeloid transforming gene binds a consensus sequence GAAGATGAG. Oncogene. 5:925-927.

8. Morishita, K., Parganas, E., Matsugi, T., and Ihle, J.N. 1992. Expression of the EVI1 zinc finger gene in $32 \mathrm{Dcl} 3$ myeloid cells blocks granulocytic differentiation in response to granulocyte colony-stimulating factor. Mol. Cell. Biol. 12:183-189.

9. Kreider, B.L., Orkin, S.H., and Ihle, N.J. 1993. Loss of erythropoietin responsiveness in erythroid progenitors due to the expression of the EVI1 myeloidtransforming gene. Proc. Natl. Acad. Sci. U. S. A. 90:6454-6458.

10. Sitailo, S., Sood, R., Barton, K., and Nucifora, G. 1999.
Forced expression of the leukemia-associated gene EVI1 in ES cells: model for myeloid leukemia with 3 q26 rearrangements. Leukemia. 13:1639-1645.

11. Louz, D., et al. 2000. Erythroid defects and increased retrovirally-induced tumor formation in Evi1 transgenic mice. Leukemia. 14:1876-1884.

12. Chi, Y., Senyuk, V., Chakraborty, S., and Nucifora, G. 2003. EVI1 promotes cell proliferation by interacting with BRG1 and blocking the repression of BRG1 on E2F1 activity. J. Biol. Chem. 278:49806-49811.

13. Barjesteh van Waalwjik van Doorn-Khosrovani, S., et al. 2003. High EVI1 expression predicts poor survival in acute myeloid leukemia: a study of 319 de novo AML patients. Blood. 101:837-845.

14. Alessandrino, E.P., et al. 2001. Myelodysplastic syndromes: recent advances. Haematologica. 86:1124-1157.

15. Martinelli, G., et al. 2003. Association of 3q21q26 syndrome with different RPN1/EVII fusion transcripts. Haematologica. 86:1221-1228.

16. Russell, M., et al. 1994. Expression of EVI1 in myelodysplastic syndromes and other hematologic malignancies without 3q26 translocations. Blood. 84:1243-1248.

17. Greenberg, P.L., Young, N.S., and Gattermann, N. 2002. Myelodysplastic syndromes [review]. Hematology (Am. Soc. Hematol. Educ. Program). 136-161.

18. Parker, J.E., and Mufti, G.J. 2004. The myelodysplastic syndromes: a matter of life or death. Acta Haematol. 111:78-99.

19. Matsushima, T., et al. 2003. Prevalence and clinical characteristics of myelodysplastic syndrome with bone marrow eosinophilia or basophilia. Blood. 101:3386-3390.

20. Kurzrock, R., Cortes, J., and Kantarjian, H. 2002. Clinical development of farnesyltransferase inhibitors in leukemias and myelodysplastic syndrome. Semin. Hematol. 39:20-24.

21. List, A.F. 2002. New approaches to the treatment of myelodysplasia. Oncologist. 7:39-49.

22. Mufti, G., List, A.F., Gore, S.D., and Ho, A.Y. 2003. Myelodysplastic syndrome. Hematology (Am. Soc. Hematol. Educ. Program). 176-199.
23. List, A., et al. 2003. Opportunities for Trisenox (arsenic trioxide) in the treatment of myelodysplastic syndromes. Leukemia. 17:1499-1507.

24. Cantor, A.B., and Orkin, S.H. 2002. Transcriptional regulation of erythropoiesis: an affair involving multiple partners. Oncogene. 21:3368-3376.

25. Wu, H., Liu, X., Jaenish, R., and Lodish, H.F. 1995. Generation of committed erythroid and BFU-E and CFU-E progenitors does not require erythropoietin or the erythropoietin receptor. Cell. 83:59-68.

26. Murone, M., Carpenter, D.A., and De Sauvage, F.J. 1998. Hematopoietic deficiencies in c-mpl and TPO knockout mice. Stem Cells. 16:1-6.

27. Mignotte, V., et al. 1994. Structure and transcription of the human c-mpl gene (MPL). Genomics. 20:5-12.

28. Hawley, R.G., Lieu, F.H., Fong, A.Z., and Hawley, T.S. 1994. Versatile retroviral vectors for potential use in gene therapy. Gene Ther. 1:136-138.

29. Wagner, K.U., et al. 2000. Conditional deletion of the Bcl-x gene from erythroid cells results in hemolytic anemia and profound splenomegaly. Development. 127:4949-4958.

30. Senyuk, V., et al. 2002. The leukemia-associated transcription repressor AML1/MDS1/EVI1 requires $\mathrm{CtBP}$ to induce abnormal growth and differentiation of murine hematopoietic cells. Oncogene. 21:3232-3240.

31. Cuenco, G.M., Nucifora, G., and Ren, R. 2000. Human AML1/MDS1/EVI1 fusion protein induces an acute myelogenous leukemia (AML) in mice: a model for human AML. Proc. Natl. Acad. Sci. U. S. A. 97:1760-1765.

32. Buonamici, S., et al. 2002. Real-time quantitation of minimal residual disease in inv(16)-positive acute myeloid leukemia may indicate risk for clinical relapse and may identify patients in a curable state. Blood. 99:443-449.

33. Nucifora, G., et al. 1994. Consistent intergenic splicing and production of multiple transcripts between $A M L 1$ at $21 \mathrm{q} 22$ and three unrelated genes at $3 \mathrm{q} 26$ in the $(3 ; 21)$ (q26;q22) translocation. Proc. Natl. Acad. Sci. U. S. A. 91:4004-4008. 